Urología Neurológica y Urodinámica

Arch. Esp. Urol. 2010; 63 (5): 355-361

\title{
CISTOCELES DE ALTO RIESGO URODINÁMICO
}

Jesús Salinas Casado, Santiago Méndez Rubio, Miguel Virseda Chamorro, Humberto Pelaquim y Ángel Silmi Moyano.

Servicio de Urología. Hospital Clínico de San Carlos. Universidad Complutense de Madrid. España.

Resumen.- OBJETIVO: Comprobar la acomodación vesical de una serie de cistoceles remitidos para estudio urodinámico.

MÉTODOS: Se realizó un estudio retrospectivo de una serie de pacientes con cistocele, sometidos a historia clínica, estudio vídeo-urodinámico, RMN del suelo pélvico y tracto urinario inferior, ecografía urológica y cistoscopia. Se excluyeron los casos con disfunción neurógena e infección urinaria. La terminología siguió los criterios de la ICS, cuando no se especifica lo contrario. La serie comprende a 3.333 casos de cistocele de los que 616 casos corresponden a cistoceles grado III. Los casos

\section{CORRESPONDENCIA}

Jesús Salinas Casado

Servicio de Urología

Hospital Clínico de San Carlos

Profesor Martín Lagos s/n

28040 Madrid (España)

jsalinascasado@yahoo.es

santiago.mendez@telefonica.net

Aceptado para publicar: 12 de junio 2009 con acomodación vesical disminuida correspondieron a 3 casos, esto es el 0,009\% del total 11 por mill y el 0,5\% de los cistoceles grado III (1 por 200)

RESULTADOS: Todos los casos de cistocele de baja acomodación, se asociaron a sensación de bulto en vagina y síntomas funcionales del tracto urinario inferior. Ninguna refería incontinencia urinaria a la tos. En estas pacientes también se demostraron alteraciones urodinámicas de la fase miccional tipo hipo/acontractilidad del detrusor y residuo postmiccional. Las pacientes fueron sometidas a distintas técnicas vaginales y abdominales de corrección del cistocele (con cirugía anti-incontinencia preventiva), obteniendo una desaparición del bulto en vagina, mejoría de los síntomas funcionales del tracto urinario inferior, con normalización de la acomodación vesical y contractilidad del detrusor, y con desaparición del residuo postmiccional.

CONCLUSIONES: Aunque no son frecuentes los cistoceles de alto riesgo, habrá que descartarlos ante cistoceles de alto grado, que aparte de la baja acomodación vesical, presentan un detrusor hipo/acontráctil y residuo postmiccional. La cirugía correctora del cistocele, no sólo reduce el prolapso y mejora los síntomas funcionales del tracto urinario inferior, sino que normaliza las alteraciones urodinámicas.

Palabras clave: Cistocele. Acomodación vesical disminuida. Cistocele alto riesgo.

Summary.- OBJECTIVES: To assess the bladder compliance in a series of cystoceles referred for urodynamic study.

METHODS: Retrospective study of a series of patients with cystocele undergoing medical history, videurodynamic study, pelvic MRI and lower urinary tract, urological 
ultrasound and cystoscopy. We Excluded cases with neurogenic dysfunction and urinary infection. The terminology followed the criteria of the ICS, if not specified otherwise. The series includes 3333 cases of cystocele 616 of which are grade III cystocele. There were 3 cases with low bladder compliance; this is $0.0009 \%$ of total (1:1000) and 0.5\% of grade III cystocele (1:200)

RESULTS: All cases of cystocele whit low compliance were associated with feeling of a bulk in the vagina and functional symptoms of lower urinary tract (LUTS). No urinary incontinence was related to cough. These patients also showed urodynamic alterations in the voiding phase, type hypo / acontractile detrusor and postvoid residual. The patients were subjected to various techniques of abdominal and transvaginal cystocele repair (with preventive anti-incontinence surgery), getting a vagina bulk disappearance, improvement of symptoms of lower urinary tract function, normalization of bladder compliance and detrusor contractility, with elimination of the postvoid residual.

CONCLUSIONS: Although they are not frequent, high-risk cystoceles should be discarded in high-grade cystocele that apart from low bladder accommodation, have a hipo/acontractile detrusor and postvoid residual. Surgical correction of cystocele not only reduces the bulk and LUTS, but normalizes urodynamic alterations.

Keywords: Cystocele. Low bladder compliance. High risk cystocele.

\section{INTRODUCCIÓN}

El diagnóstico de cistocele, se asocia frecuentemente a una alteración de la fase de llenado vesical en la forma de incontinencia urinaria, y menos frecuentemente a alteraciones de la fase de vaciamiento, tipo retención urinaria crónica. Los datos urodinámicos relacionados con los desórdenes de la fase de vaciamiento corresponden generalmente a detrusor hipo/acontráctil y obstrucción del tracto urinario inferior (1). Los datos urodinámicos relacionados con los desórdenes de la fase de llenado vesical corresponden generalmente a hiperactividad del detrusor e incontinencia urinaria de esfuerzo (2).

No obstante, en ocasiones, los síntomas funcionales del tracto urinario inferior en la mujer, descritos anteriormente, se asocian a una acomodación vesical disminuida durante la cistomanometría, lo que supone un alto riesgo dinámico para el tracto urinario superior de estas pacientes, a semejanza de lo que ocurre en otras patologías como: disfunción neurógena vésicouretral, cistopatías...

El objetivo de esta publicación, iría dirigido a presentar estos casos, así como destacar su importancia pronóstica y terapéutica.

\section{MATERIAL Y MÉTODOS}

Se realizó un estudio retrospectivo de una serie de casos con diagnóstico de cistocele que fueron sometidos a estudio vídeo-urodinámico (flujometría con residuo postmiccional, cistomanometría, y test presión detrusor/flujo miccional), asociado a estudio radiológico del tracto urinario inferior. A las pacientes se les realizó así mismo una anamnesis y exploración física, y se completó el estudio con ecografía renovesical, cistoscopia y resonancia magnética nuclear del tracto urinario inferior y suelo pélvico. La terminología siguió los criterios de la ICS (Internacional Continence Society) (3) si no se especifica lo contrario (Adot et al. 2005, Salinas et al. 2005).

Se excluyeron los casos de disfunción neurógena vésicouretral e infección urinaria.

Esta serie comprendió 3.333 casos de cistocele, de los que 616 casos correspondieron a cistocele grado III. Los casos de cistocele con acomodación vesical disminuida correspondieron a 3 casos, esto es el 0,0009\% del total de cistocele (uno por mil) y el $0,5 \%$ de los cistoceles grado III ( 1 por 200).

\section{RESULTADOS}

\section{Caso no 1}

Mujer de 61 años de edad. Historia de 5 años de sensación de bulto en vagina. Síntomas funcionales del tracto urinario inferior tipo vaciamiento (dificultad miccional y calibre miccional disminuido); no incontinencia urinaria. Histerectomía abdominal hace 4 años.

La cistomanometría demostró una acomodación vesical disminuida (presión elevada detrusor al llenado $45 \mathrm{~cm} \mathrm{H} 2 \mathrm{O}$ ). El estudio detrusor/flujo miccional demostró una afectación de la contractilidad isotónica del detrusor, y residuo postmiccional (Figura 1).

El estudio radiológico demostró un cistocele grado III (Figura 1). La RMN del suelo pélvico y tracto urinario inferior comprobó la existencia de un cistocele III, así como discreto prolapso de cúpula. La 


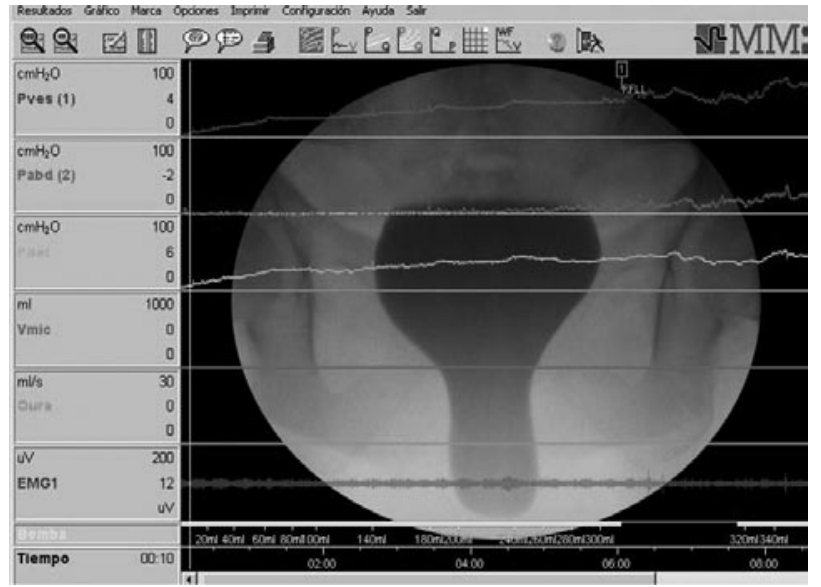

FIGURA 1. Videourodinámica preoperatoria.

Cistocele grado III. Baja acomodación vesical.

ecografía renal y vesical no reveló dilatación a nivel renal o del tracto urinario superior.

La enferma fue sometida a colposacropexia abdominal y colposuspensión técnica Ramírez (preventiva), desapareciendo la sensación de bulto en vagina, así como los síntomas funcionales del tracto urinario inferior, tipo vaciamiento.

La acomodación vesical en la cistomanometría se normalizó (presión detrusor al llenado de 1 $\mathrm{cm} \mathrm{H2O}$ ) (Figuras 2 y 3). La capacidad contráctil del detrusor se normalizó en el test presión detrusor/flujo miccional, desapareciendo el residuo postmiccional. Los estudios morfológicos del tracto urinario inferior y suelo pélvico demostraron la corrección de los prolapsos pélvicos (Figuras 2 y 4 ).

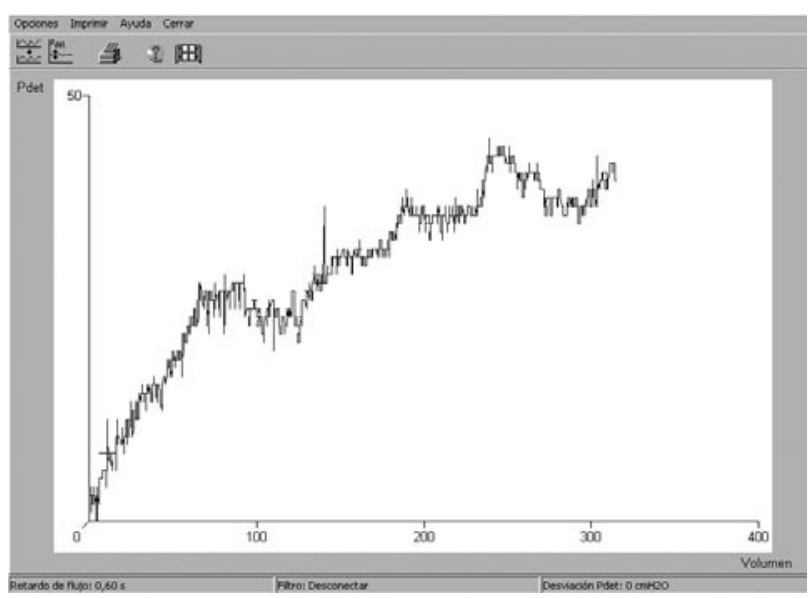

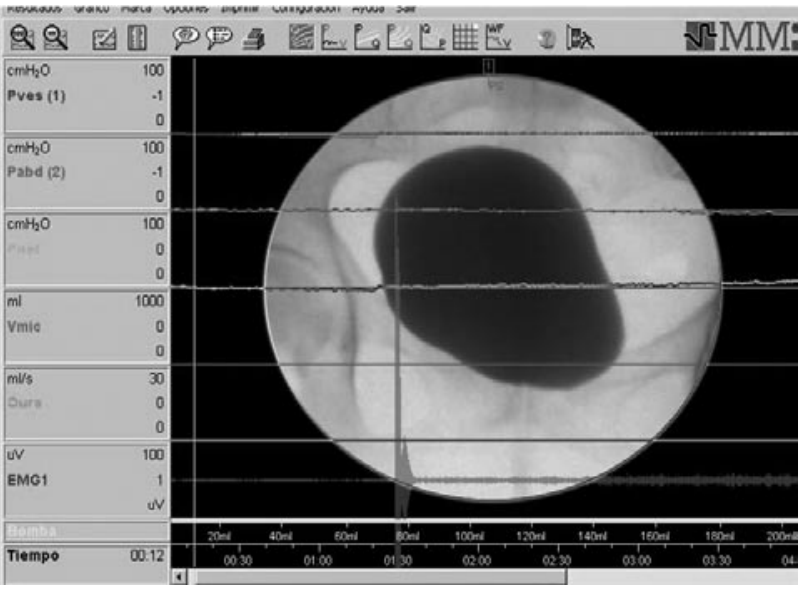

FIGURA 2. Videourodinámica postcirugía. Acomodación vesical normal.

\section{Caso $n^{\circ} 2$}

Mujer de 76 años de edad. Historia de dos años de sensación de bulto en vagina asociado a dificultad miccional y calibre miccional disminuido (síntomas funcionales del tracto urinario inferior tipo vaciamiento). No incontinencia urinaria. La cistomanometría demostró una acomodación vesical disminuida, con una presión elevada del detrusor al llenado de $40 \mathrm{~cm} \mathrm{H} 20$. El test presión detrusor/flujo miccional demostró un detrusor acontráctil.

El estudio radiológico demostró un cistocele grado III. La RMN del suelo pélvico y tracto urinario inferior reveló el cistocele grado III, así como prolapso uterino grado III y rectocele III. La ecografía renovesical fue normal, no apreciándose dilatación ureteral.

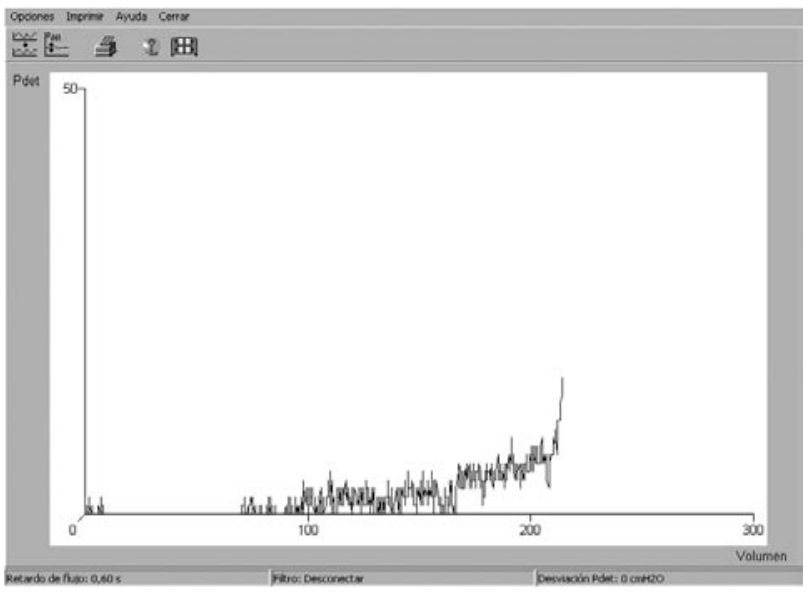

FIGURA 3. Acomodación vesical pre y postoperatoria. 
La enferma fue sometida a histerectomía vaginal, malla anterior 4 anclajes (Perigee), malla posterior 2 anclajes (Apogee) y "Needleless" preventivo. En el estudio postcirugía la enferma refirió ausencia de bulto en vagina, así como calibre miccional normal y desparición de la dificultad miccional.

La cistomanometría demostró una normalización de la acomodación vesical (presión detrusor llenado $1 \mathrm{~cm} \mathrm{H} 20$ ), y la demostración en el test presión detrusor/flujo miccional de una micción por contracción (involuntaria) del detrusor, con mínimo residuo postmiccional (no significativo).

Los estudios morfológicos del tracto urinario inferior y suelo pélvico demostraron una gran reducción de los prolapsos pélvicos.

\section{Caso no 3}

Mujer de 69 años de edad. Historia antigua de sensación de bulto en vagina. Síntomas funcionales del tracto urinario inferior de almacenamiento, tipo urgencia-incontinencia. No incontinencia urinaria a la tos.

El estudio urodinámico demostró una presión del detrusor de llenado elevada de $30 \mathrm{~cm} \mathrm{H} 20$ y una micción con prensa abdominal (detrusor acontráctil) y residuo postmiccional.

El estudio morfológico del tracto urinario inferior demostró un cistocele grado III, que fue comprobado mediante Resonancia Magnética Nuclear
(RMN) del suelo pélvico y tracto urinario inferior. Mediante esta última técnica se comprobó la presencia así mismo de un prolapso uterino grado II.

Tanto la ecografía renovesical, como la cistoscopia fueron absolutamente normales.

La enferma fue sometida a colporrafia anterior, "Needleless" (preventiva) y Manchester, despareciendo la sensación de bulto en la vagina. Se mantuvo la polaquiuria y urgencia-incontinencia.

El estudio urodinámico demostró una normalización de la presión del detrusor de llenado $(2 \mathrm{~cm}$ H2O), y una micción con contracción del detrusor (involuntaria) y ausencia de residuo postmiccional.

Los estudios morfológicos del tracto urinario inferior y el suelo pélvico demostraron una corrección de los prolapsos pélvicos.

\section{DISCUSIÓN}

Cuando se habla de cistocele de alto riesgo, se refiere al riesgo urodinámico de repercusión nociva sobre el tracto urinario superior, y no al riesgo de recidiva del prolapso post-cirugía correctora, ya que algunos autores (Gardy et al.) (4) proponían una cirugía del cistocele, cuando el cistocele era de bajo grado y su correción más efectiva, antes de que

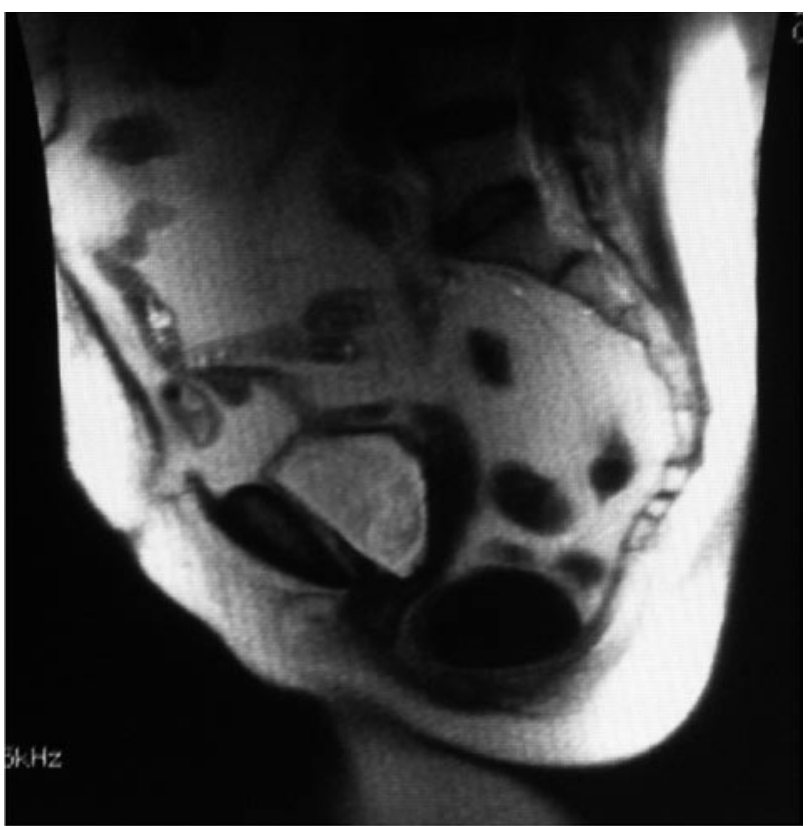

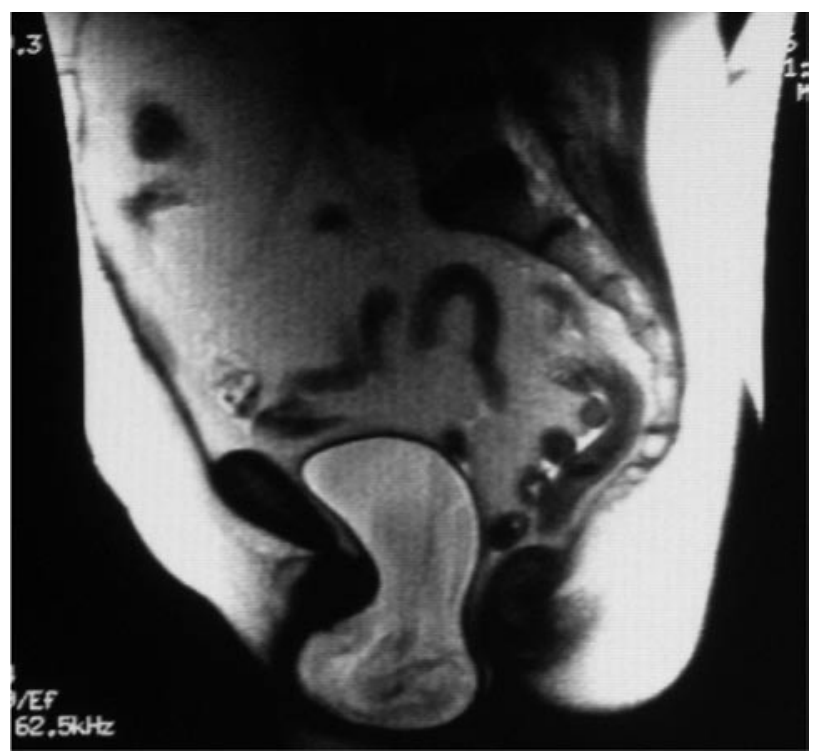

FIGURA 4A). Pre-cirugía. Se demuestra Cistocele grado III y discreto prolapso de cúpula. 4B). Post-cirugía. Se demuestra corrección prolapsos pélvicos. 
adquirieran, con el transcurso del tiempo, grados mayores, basándose en la evolución natural del crecimiento progresivo del cistocele, lo que no siempre se produce.

Se han descrito casos de ureterohidronefrosis en cistoceles de alto grado (Delaere) (5) que se han explicado por la tracción ureteral en dirección caudal producidos por el efecto arrastre del prolapso vesical.

A pesar de la poca frecuencia de estos cistoceles de baja acomodación, habrá que tenerlos presentes ante la comprobación por técnicas de imagen de dilataciones del tracto urinario superior. En ocasiones podrían corresponder no a tracciones ureterales producidas por el prolapso pélvico (sobre todo en el trígonocele), sino a la repercusión de una baja acomodación vesical sobre los riñones y tracto urinario superior, a semejanza de los que ocurre en otras condiciones como las vejigas neurógenas, disfunciones vésicouretrales en el niño, obstrucción del tracto urinario inferior en el varón, algunas cistopatías, sonda vesical permanente... De ahí la necesidad de realizar ecografía del tracto urinario superior ante la sospecha de estos cistoceles de alto riesgo.

No obstante, en nuestros casos no se demostró dilatación del tracto urinario superior, posible-

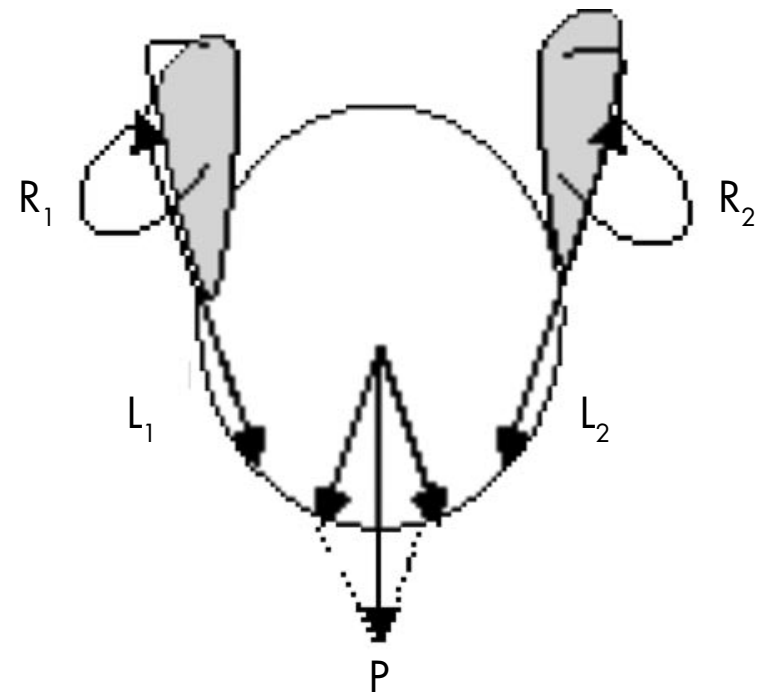

FIGURA 5. Modelo de cistocele precirugía. La vejiga se mantiene suspendida de la pelvis por un ligamento a cada lado que soportan y transmiten el peso de la vejiga $(P)$, descompuesto en 2 fuerzas, $L 1$ y $L 2$, a las paredes vesicales (R 1 y $R 2$ ). mente, porque la baja acomodación vesical sería de reciente instauración.

Por otra parte, hubiera sido interesante realizar telecistomanometría (6), a fin de comprobar si la baja acomodación vesical precirugía en el estudio urodinámico standard, se confirmaba en el estudio ambulatorio, o correspondía a una hiperactividad del detrusor.

Apoyando nuestros resultados, Long et al. (7) refieren una mejor acomodación vesical en su grupo control, cuando lo comparan a su grupo de prolapsos pélvicos severos grados III/IV.

La explicación biomecánica de los cistoceles de baja acomodación, se entendería partiendo de un modelo de vejiga que se encuentra suspendida en la pelvis por un ligamento (o conjunto de ligamentos), uno a cada lado. El peso de la vejiga $(P)$ se puede descomponer en 2 fuerzas ( $L 1$ y L2), que son las que soportan los ligamentos de los que está suspendida la vejiga. Estas dos fuerzas por el principio de acción y reacción (tercera ley de Newton) originan en la pared vesical otras 2 fuerzas iguales y de sentido contrario (R 1 y R2). Las dos fuerzas de reacción de la pared vesical originan una tensión en el seno de la pared (Figura 5). Según la ley de Laplace, esa tensión de la pared (T), origina una presión en el interior de la vejiga (Pvesical), directamente proporcional al doble de la tensión de su pared (2T) e inversamente proporcional al radio de la vejiga (Pvesical=2T/R).

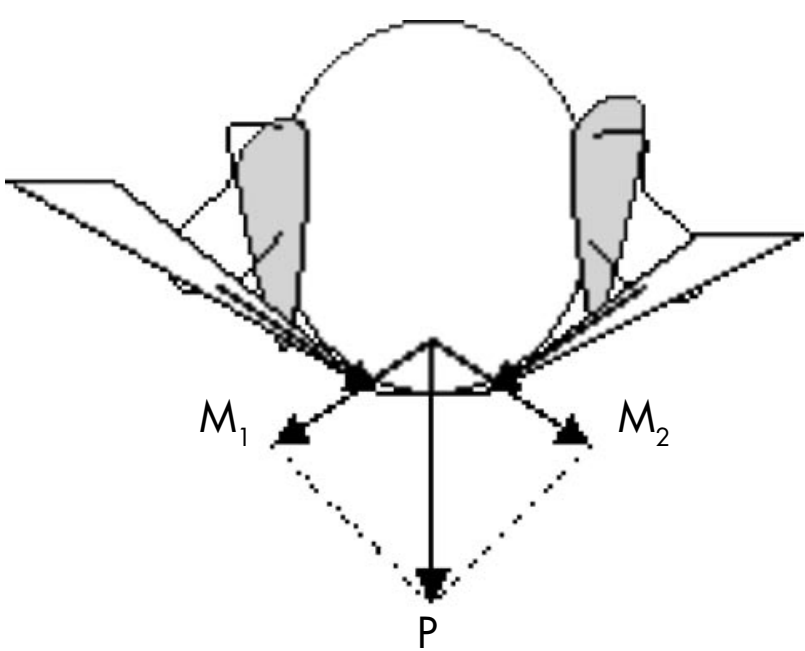

FIGURA 6. Modelo de vejiga post-corrección del cistocele. La implantación de una malla vesical, supone que ésta soporta el peso de la vejiga (MI y M2) y por lo tanto libera la tensión de las paredes vesicales. 
Durante el llenado vesical, la acomodación (C) se define como la relación entre el incremento del volumen $(\Delta V)$ y el incremento de la presión $(\Delta P)$ $\mathrm{C}=\Delta \mathrm{V} / \Delta \mathrm{P}$. Al producirse un aumento de la presión vesical por el aumento de su tensión, la acomodación vesical disminuirá.

Desde el punto de vista biológico, estas vejigas de baja acomodación, más que suspendidas en la pelvis por unos ligamentos, están fijadas por dichos ligamentos, bien debido a fibrosis, rigidez, roturas, ya que, de presentar unos elementos de sostén suficientemente elásticos no se pondrían en marcha estos mecanismos descritos. La reducción del cistocele (generalmente la colocación de una malla) origina una redistribución de las fuerzas resultantes del peso de la vejiga, que pasan a ser soportadas por la malla, disminuyendo la tensión de las paredes vesicales, y por tanto, mejorando su acomodación.

En nuestros casos, todas las técnicas utilizadas en la corrección del cistocele y otros prolapsos tanto vaginales como abdominales), normalizaron la acomodación vesical.

Se podría comparar la acomodación vesical, ante la existencia de prolapsos pélvicos en otros compartimentos, con ausencia de cistocele. No obstante, este estudio es difícil llevarlo a cabo en la clínica, dada la casi constante asociación de los prolapsos en los distintos compartimentos, al tratarse en la mayoría de los casos de una disfunción global del suelo pélvico.

Aunque ningún caso presentó incontinencia urinaria a la tos, en todos los casos se realizó una cirugía anti-incontinencia preventiva. La asociación de una técnica anti-incontinencia a la corrección del cistocele de alto grado como aconsejaría Mourtzinos et al 2006 (8), no interfirió con la obtención de unos buenos resultados, referidos a la normalización de la acomodación vesical, a pesar de que en algunas condiciones patológicas con producción de estenosis a nivel uretral $(9,10)$ (valvas uretra posterior en el niño, mielodisplasias con estenosis a nivel de uretra membranosa, estenosis uretra membranosa post-prostatectomía, post-implante esfínter artificial...) se asocian no infrecuentemente a vejigas de baja acomodación.

Por otra parte, las técnicas de corrección del cistocele, no sólo corrigieron la acomodación vesical, sino que mejoraron la contractilidad del detrusor durante la micción eliminando el residuo postmiccional.

Utilizando el mismo modelo anterior, también se puede explicar la recuperación de la contractilidad vesical con la corrección del cistocele.
La ley de Starling indica que el estiramiento de las fibras musculares se traduce en contractilidad, pero si este estiramiento excede cierto límite, la fibra muscular pierde la capacidad contráctil.

En este sentido, el estiramiento de las fibras musculares de la pared vesical debido a la fuerza de la gravedad (o presión hidrostática) origina una deformidad plástica de las fibras musculares que afecta su capacidad contráctil. La intervención del cistocele corrige esta deformidad plástica al pasar a soportar, la fuerza de la gravedad (M1 y M2), la malla que sostiene la vejiga, recuperándose la contractilidad vesical (Figura 6 ).

La recuperación de la contractilidad del detrusor se tradujo en la desaparición post-cirugía de los síntomas de la fase de vaciamiento como la dificultad miccional.

Las estimaciones de la dificultad miccional en las pacientes uroginecológicas se ha subestimado habitualmente. Se estimaba la prevalencia en un 14 $\%$. En el estudio de Haylen et al. (1 1) se ha encontrado una prevalencia del $39 \%$, muy superiores a las realizadas con anterioridad. Se sitúa en frecuencia después de la incontinencia urinaria de esfuerzo $(72$ $\%)$ y del prolapso pélvico (61\%). Se sitúa por delante de la vejiga hiperactiva (13\%). La dificultad miccional aumenta con la edad y el grado del prolapso.

\section{CONCLUSIÓN}

En conclusión, los cistoceles de alto riesgo, se refieren a cistoceles grado III, que se asocian a otros prolapsos pélvicos de diferentes compartimentos, en los que se demuestra una baja acomodación durante el llenado vesical de la cistomanometría, así como un detrusor hipo/acontráctil y residuo postmiccional en la fase de vaciamiento. La corrección quirúrgica del cistocele, se asoció no sólo a la desaparición del prolapso pélvico y mejoría de los síntomas funcionales del tracto urinario inferior sino a la normalización de las alteraciones urodinámicas tanto de la fase de llenado como de vaciamiento vesical.

\section{BIBLIOGRAFÍA Y LECTURAS RECOMENDADAS (*lectura de interés $y^{* *}$ lectura fundamental)}

**1. Salinas Casado J, Adot Zurbano JM, Dambros M, Vírseda Chamorro M, Ramírez Fernández JC, Moreno Sierra J, Marcos Díaz J, Silmi Moyano A. Factores de descompensación miccional y cistocele Arch Esp Urol, 2005;58(4):316-23. 
**2. Adot Zurbano JM, Salinas Casado J, Dambros M, Vírseda Chamorro M, Moreno Sierra J, Ramírez Fernández JC, Silmi Moyano A, Díaz JM. Alteraciones de la fase de llenado vesical y cistocele. Arch Esp Urol, 2005;58(4):309-15.

3. Abrams P, Cardozo L, Fall M, Griffiths D, Rosier P, Ulmsten U, van Kerrebroeck P, Victor A, Wein A; Standardisation. The standardisation of terminology of lower urinary tract function: report from the Standardisation Sub-committee of the International Continence Society. Neurourol Urodyn.2002;21(2):167-78. Comment in: Neurourol Urodyn. 2007;26(6):757-8; discussion 759-60.

4. Gardy M, Kozminski M, DeLancey J, Elkins T, McGuire EJ. Stress incontinence and cystoceles. J Urol, 1991;145(6):1211-3.

5. Delaere K, Moonen W, Debruyne F, Jansen T. Hydronephrosis caused by cystocele. Treatment by colpopexy to sacral promontory. Urol, 1984; 24(4):364-5.

6. Leva M, Prieto R, Regueiro JC et al: Urodinámica ambulatoria: aplicaciones y datos. En Urodiná- mica Clínica: Aspectos Básicos. Cap 14. p 465500. Ed J Salinas J Romero Pub. Luzán 5. Madrid 2002.

7. Long CY, Hsu SC, Sun DJ, Chen CC, Tsai EM, Su $\mathrm{JH}$. Abnormal clinical and urodynamic findings in women with severe genitourinary prolapse. Kaohsiung J Med Sci. 2002;18(12):593-7.

8. Mourtzinos A, Maher M, Sabih N, Rodríguez L, Raz S: To sling or not to sling: Should patients undergoing a grade IV cystocele repair have a prophylactic antiincontinence procedure? J Urol. 2006, Vol. 175, 4, Suppl. 295-296.

9. Ghoniem GM, Bloom DA, McGuire EJ, Stewart KL. Bladder compliance in meningomyelocele children. J Urol, 1989;141(6):1404-6.

10. Rapariz M, Salinas J, Paez A et al. Vejiga de alta presión de llenado. Urod A, 1992; (5): 115-9.

*11. Haylen BT, Krishnan S, Schulz S, Verity L, Law M, Zhou J, Sutherst J. Has the true prevalence of voiding difficulty in urogynecology patients been underestimated? Int Urogynecol J Pelvic Floor Dysfunct. 2007;18(1):53-6. Epub 2006 Apr 5. 